\title{
Interference of coronal enamel and dentin thickness and ambient light on pulse oximetry interpretation
}

\author{
Julio Almeida SILVA ${ }^{(a)}$ \\ Ana Helena Gonçalves \\ de ALENCAR ${ }^{(a)}$ \\ Larissa Emanuelle SESTARI(a) \\ Higor Almeida BARBOSA(a) \\ Patrícia Correia de SIQUEIRA(a) \\ Daniel Almeida DECURCIO(a) \\ Fernando Branco BARLETTA ${ }^{(b)}$ iD \\ Carlos ESTRELA(a) \\ (a) Universidade Federal de Goiás - UFG, \\ Faculdade de Odontologia, Departamento \\ de Ciências Estomatológicas, Goiânia, \\ GO, Brazil. \\ (b) Universidade Luterana do Brasil - Ulbra, \\ Faculdade de Odontologia, Departamento \\ de Endodontia, Canoas, RS, Brazil.
}

Declaration of Interests: The authors certify that they have no commercial or associative interest that represents a conflict of interest in connection with the manuscript.

Corresponding Author:

Daniel de Almeida Decurcio

E-mail:danieldecurcio@gmail.com

htrps://doi.org/10.1590/1807-3107bor-2020.vol34.0064

Submitted: March 5, 2020

Accepted for publication: April 8, 2020

Last revision: May 19, 2020

\begin{abstract}
This study evaluated the interference of enamel and coronal dentin thickness and ambient light in pulse oximetry interpretation, using $\mathrm{SaO}_{2}$ the human finger as a reference. Forty-two intact human permanent mandibular molars were sectioned mesiodistally in the central portion. Buccal and lingual dentin surfaces were drilled, and $4 \mathrm{~mm}, 3 \mathrm{~mm}$ and $2 \mathrm{~mm}$ enamel/dentin thicknesses were interposed between finger and device, after which $\mathrm{SaO}_{2}$ levels were measured by finger pulse oximetry. A reference device was designed to align pulse oximeter light-emitting diode, buccal surface of tooth, little finger, lingual surface of tooth and photodiode. Variables were described as means and standard deviation, and the confidence interval was reported. Repeated measures analysis of variance (ANOVA) was used to compare the groups, followed by Bonferroni correction. Student $t$ test for paired samples was used to determine presence of ambient light $(\alpha=0.05)$. Mean $\mathrm{SaO}_{2}$ level was lower at $4.0 \pm 0.2 \mathrm{~mm}$ thickness, regardless of presence or absence of ambient light (92.7\% and $89.3 \%$ ). The other thicknesses yielded values of $95.5 \%$ and $94.5 \%$ at $3.0 \pm 0.2 \mathrm{~mm}$, and $96.4 \%$ and $96.0 \%$ at $2.0 \pm 0.2 \mathrm{~mm}$ $(\mathrm{p}<0.001)$. There were significant differences between $\mathrm{SaO}_{2}$ values at $4.0 \pm 0.2 \mathrm{~mm}, 3.0 \pm 0.2 \mathrm{~mm}$ and $2.0 \pm 0.2 \mathrm{~mm}$ thicknesses, in the presence of ambient light. Mean $\mathrm{SaO}_{2}$ level in positive control was $96.3 \%$, and mean pulse rate was $69.5 \mathrm{bpm}$ in presence of ambient light; in the absence of light, these values were $96 \%$ and $70.5 \mathrm{bpm}$. Enamel and dentin thickness interfere with $\mathrm{SaO}_{2}$, regardless of presence or absence of ambient light. The lowest $\mathrm{SaO}_{2}$ levels were found for the thickest tooth samples.
\end{abstract}

Keywords: Oximetry; Dentin; Dental Enamel; Dental Pulp.

\section{Introduction}

Planning a clinical procedure starts with making the correct decision based on an appropriate diagnosis. ${ }^{1}$ The level of pulp oxygen saturation helps determine the condition of this tissue when making a clinical diagnosis of pulpal inflammation changes. Pulse oximetry based on the light spectrum and its propagation is an important diagnostic resource in endodontics. ${ }^{2}$ 
Transillumination, photoactivation of restorative materials, laser use for cleaning infected root canals, and biomodulation before and after an operation are resources applied to diagnose and treat hard tissue lesions. Dentistry uses light at several spectral bands, but its use as a quantitative optical method to make endodontic diagnoses requires knowing how it propagates through dental structures. ${ }^{3}$ When dental tissues receive the emission of red light (spectrum of about $660 \mathrm{~nm}$ ), the basic principles of interaction between light and matter are in place, and reflectance, transmittance, absorbance and diffraction occur. ${ }^{4}$ Reflectance is characterized by radiation scattering on a surface, with no change in frequency or monochromatic components, and may be total or partial. Transmittance refers to the intensity of radiation that propagates through a solid, liquid or gaseous material, with no change in frequency or monochromatic components, and each material may have a higher or lower transmittance index. Absorbance is the measure of loss in intensity of the principal light ray, the base-10 logarithm of the ratio between incident and transmitted light, without considering other effects, such as diffraction and light scattering. Diffraction occurs because electromagnetic radiation can move around objects, depending on the wavelength range of the light, and because of interferences, which deflect the principal beam. The intensity of these phenomena may range substantially, depending on the type of material. ${ }^{4}$

Pioneering studies on the optical properties of hard dental tissues began in the $1970 \mathrm{~s}, 5,6$ and later studies have established that enamel prisms diffract light. ${ }^{7}$ Different transmittance results have been achieved when the light source is positioned perpendicular or at an angle to the enamel surface. ${ }^{3}$ In dentin, dentinal tubules are light scattering agents, and the diffraction in these tubules is greater than that found in enamel. . $^{6,8} 9$

The thickness of the dental substrate, whether enamel or dentin, seems to play an important role in the degree of attenuation of the transmitted wavelengths, but its effect is not linear. Variations of about $30 \%$ in transmittance between two samples with the same thickness have already been described. ${ }^{9}$ The vector of incident light seems to be important in light transmittance, depending on the spectral band. Studies have found changes in light transmittance according to tooth position. ${ }^{8,9,10}$ Trials with dentin disks found that light propagates better through hydrated than dehydrated disks. ${ }^{8,11}$

Clinical studies with intact teeth using a pulse oximeter have found variations in the mean levels of oxygen saturation in the dental pulp, at levels that are all below those of the patient's finger. ${ }^{12}$ Pulp vitality is usually an indication of integrity of vascular supply to the dental pulp. ${ }^{13}$ Blood flow is greater in the pulp than in other oral tissues, and is similar to the levels found in the brain. The greatest capillary density is found in the peripheral layer of the coronal portion. ${ }^{14}$ The mean level of pulp oxygen saturation ranges from $79.31 \%{ }^{2}$ to $94 \%{ }^{15}$ in maxillary central incisors, from $78.51 \%{ }^{16}$ to $87.47 \%{ }^{17}$ in maxillary central incisors, from $79.85 \%{ }^{2}$ to $91 \%{ }^{12}$ in canines, and is $86.2 \%$ in premolars, ${ }^{18} 83.59 \%$ in maxillary molars, and $86.89 \%$ in mandibular molars. ${ }^{19}$

The variations relative to the position of the pulp surrounded by hard tissues, associated with enamel and dentin thickness and with the volume of the pulp chamber seem to be responsible for the lower pulse oximetry values for the pulp of intact teeth. ${ }^{20}$ Age has been associated with a gradual reduction in oxygen saturation..$^{18}$ Clinical studies with inflamed pulps $^{13,21}$ have found oxygen saturation values of $87.4 \%$ and $85.4 \%$ for reversible pulpitis, $83.1 \%$ and $81.6 \%$ for irreversible pulpitis, and $74.6 \%$ and $70.7 \%$ for pulpal necrosis. It should be considered that pulp conditions are adverse in traumatic dental injuries. ${ }^{22}$

A study found that mean general oxygen saturation levels in the dental pulps of premolars were $86.2 \%$ (range: 69.0 to 99.0), hence below those found in the index finger of the same patients (mean: $93.7 \%$; range: 70.0 to 99.0$)$. Mean oxygen saturation of the index finger was $92.89 \%$. There were no significant correlations between oxygen saturation levels in molars (85.09\%) and in the index finger $(92.88 \%){ }^{19}$

Several factors may interfere with the measurement of oxygen saturation, such as dental enamel thickness and presence of light. This study evaluated the interference of coronal enamel and dentin thickness in the interpretation of pulse oximetry, using finger oxygen saturation as a reference. 


\section{Methodology}

\section{Sample selection}

The sample comprised mandibular molars extracted for prosthetic or periodontal reasons from male and female patients aged 18 to 40 years, seen in the Dental Urgency Service of the School of Dentistry of the Federal University of Goiás, Brazil. This experimental study was approved by the Research Ethics Committee of the Federal University of Goiás, Goiânia, Brazil, according to protocol CAAE 90795318.7.00005083.

After extraction, the teeth were immersed in 5.0\% sodium hypochlorite (Fitofarma, Lt. 20442, GO, Brazil) for 30 minutes, and then cleaned and stored in a $0.2 \%$ thymol solution.

A sample of 42 teeth was needed to estimate values at a $90 \%$ confidence interval $(\alpha=0.05)$, and detect a difference of $5 \%$ between the results of oxygen saturation levels using a pulse oximeter (Smiths Medical PM, Waukesha, USA) and three thicknesses of dental material. The initial sample had 59 teeth, with an estimated $20 \%$ reduction due to exclusion criteria and loss of $20 \%$ during preparation of the surfaces. This study included intact mandibular first and second molars. After visual examination, the teeth with morphological coronal abnormalities, stains, cracks, or enamel fractures were excluded, as well as those with a buccal or lingual thickness smaller than $4.0 \mathrm{~mm}$, determined after sectioning and finding evidence of coronal dentin color changes, caries, or pulp chamber atresia.

Mesiodistal, buccolingual and occlusocervical dimensions were measured using a digital caliper (Fowler/Sylvac, Crissier, Switzerland) calibrated to $0.01 \mathrm{~mm}$. The mesiodistal dimension was measured from the most central contact point on the mesial surface to the contralateral point on the distal surface. Afterwards, the highest cusp of the buccolingual surface was measured up to the cementoenamel junction to obtain the occlusocervical dimension. The distance between the mean point on the buccal surface and the mean point on the lingual surface was determined as the buccolingual dimension of the crown.

\section{Sample preparation}

After the buccal and lingual surfaces were identified, the teeth were sectioned mesiodistally in the central portion using a 4 " $\times 0.12 \times 0.12$ diamond wafering blade (Extec, Enfield, USA) mounted on a precision saw (Labcut 1010, Extec, London, UK) and operated under water spray at a cutting speed of $230 \mathrm{rpm}$. Samples were stored in individually numbered recipients. A digital caliper (Fowler/Sylvac, Crissier, Switzerland) was used to measure the thickness of each surface at the point of greatest thickness. As needed, the internal surface of the dentin was ground with $\mathrm{SiC}$ sandpaper (P100, Fortel Indústria e Comércio de Equipamentos Metalográficos, São Paulo, Brazil) on an automatic polisher (Politriz Lixadeira de Velocidade Variável, PVV, Fortel, São Paulo, Brazil), under water spray, at $230 \mathrm{rpm}$, to standardize thicknesses to $4.0 \mathrm{~mm}$. Two measurements were made to calculate a mean value for the thickness of each surface. Samples were cleaned with pumice and water using a Robson brush (Microdont, São Paulo, Brazil) at low rotation to remove debris and surface stains. After measuring the 0.4 -mm surfaces with the oximeter, the surfaces were sanded to a thickness of $3.00 \mathrm{~mm}$, according to the protocol previously described. The oximetry was performed, after which the specimens were drilled again to a thickness of $2.00 \mathrm{~mm}$, and the oximetry results were recorded. The sections were kept hydrated during the entire experiment.

\section{Participant recruitment}

A volunteer participant was recruited before the study began, was informed about the study protocol and its importance, and signed an informed consent term. The participant inclusion criteria were age 18 to 26 years and mean oxygen saturation $\geq 95 \%$. The exclusion criteria were extremely white skin (pale), dark skin, smoking, pregnancy, history of vascular or cardiovascular disease, and use of systemic medication.

\section{Pulse oximetry}

This study was conducted using a portable pediatric pulse oximeter, model 3301 (Smiths Medical PM Inc., Waukesha, USA) (Figure A), with a Universal Y 3043 finger sensor, $660 \mathrm{~nm}$ red light $(2 \mathrm{~mW})$ and 
$905 \mathrm{~nm}$ infrared light (2.0-2.4 mW) (Figure B). A specially designed device was used as a reference during this trial, to ensure that the light emitting diode and the photodiode of the pulse oximeter, the participant's finger and the sample were all aligned, that movements were avoided and that the sensors were kept in position by the operator. The device was manufactured by taking impressions of the light emitting diode and the photodiode, and then defining where the dental surface had to be held on each impression. Afterwards, the impressions were juxtaposed in a portable vise to ensure standardization of the point of oximetry measurement (Figure C).

The pulse oximeter sensors were then fixed to the device at the predetermined points. The study participant was sitting while the measurements were taken, with his arm on a table. The volunteer was told not to move during measurements. After confirmation that the participant was not wearing nail polish on his little finger, his finger was positioned to measure his oxygen saturation (\%) and pulse rate (bpm) without interposing the dental structure; these measurements were defined as the positive control. Oxygen saturation was measured twice, the first time 30 seconds after the sensor was placed on his finger, and the second time, 30 seconds later. Room temperature was kept at $24^{\circ} \mathrm{C}\left( \pm 1^{\circ} \mathrm{C}\right)$ during measurements, and ambient light was provided by the LED bulbs (18 w) (Ourolux Led Bulb, São Paulo, Brazil) on the ceiling of the laboratory. Afterwards, the entire device was covered and the result of oximetry and pulse rate were recorded in the absence of light according to the previously described protocol. Oxygen saturation and pulse rate for the positive control group were taken before evaluating the samples in each thickness group.

Afterwards, the buccal and lingual surfaces were removed from the storage solution, and the excess liquid was dried with a paper towel. The buccolingual surface was placed between the participant's fingernail and the light emitting diode, so that the light reached the thickest point, and the lingual surface was placed between the finger pulp and the photodiode of the pulse oximeter (Figure C). The negative control of the trial was obtained by juxtaposing the buccal surface under the light emitting diode and the lingual surface under the photodiode, without juxtaposing the participant's little finger.

Oximetry measurement of each sample lasted about 5 minutes, thus ensuring that the samples remained hydrated. Pulse oximetry was measured in the little finger of the participant's right hand in half of the samples of each group, and in that of his left hand in the other half, so that the sensor remained placed intermittently and for less than two hours on each finger. This ensured that there would be no risk to the participant.

The data were collected by a single observer, experienced in pulse oximetry measurements, and blinded to the results. Another participant recorded
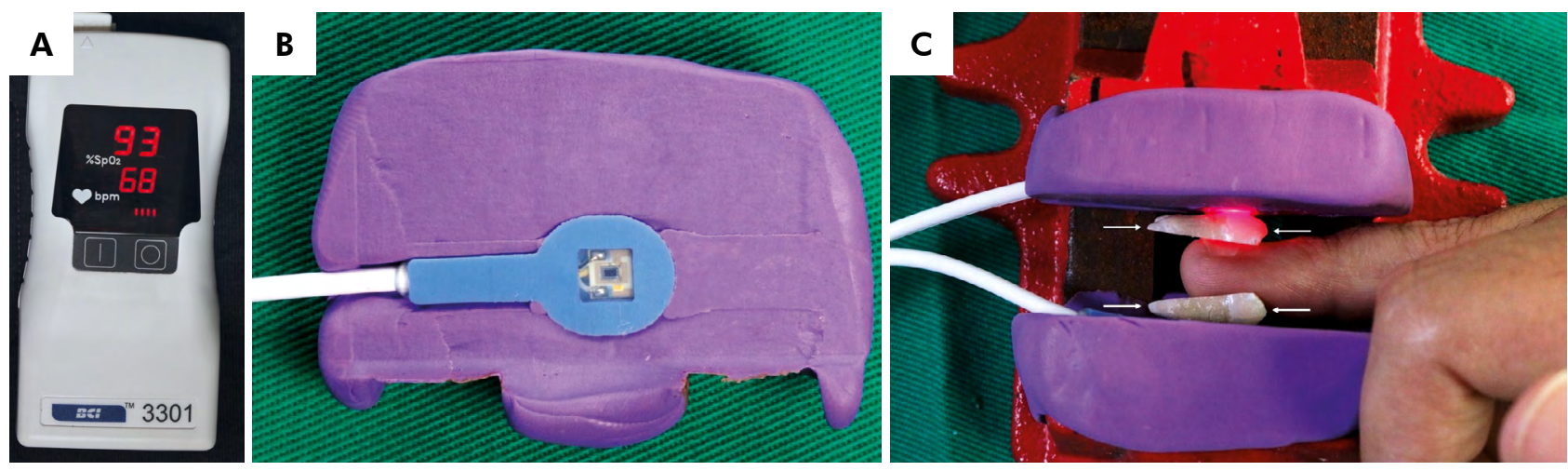

Arrows indicate the dental surfaces interposed between the participant's finger and the light emitting diode and the photodiode.

Figure. Portable pediatric pulse oximeter scoring 93\% oxygen saturation and 68 beats per minute (A). Universal Y 3043 finger sensor adapted to take impressions (B). Impressions juxtaposed in a portable vise (C). 
the pulse oximeter readings, so that the observer saw the values only at the end of the trial.

\section{Statistical analysis}

All the data were entered in an Excel spreadsheet and later exported to SPSS 20.0 software (SPSS 20.0 for Windows, Chicago, USA, 2011) for statistical analysis. Variables were described as means and standard deviations. The confidence interval for the difference between means was calculated, and repeated measures analysis of variance (ANOVA) was used to compare the groups, followed by the Bonferroni correction for multiple comparisons. The Student $t$ test for paired samples was used to compare mean oxygen saturation levels within the groups in the presence and absence of light. The level of significance was set at $5 \%$ for all comparisons.

\section{Results}

Oxygen saturation and pulse rate were measured twice in the presence and twice in the absence of ambient light for each thickness group. The two measurements were compared, and no statistically significant differences were found, thus allowing the means of the two values to be used to perform the analyses $(p>0.05)$.

Table shows the mean oxygen saturation values (\%) in each thickness group, both in the presence and absence of ambient light, revealing that there were statistically significant differences among the groups. The mean oxygen saturation was lower at $4.0 \pm 0.2 \mathrm{~mm}$, than at $3.0 \pm 0.2 \mathrm{~mm}$ or at $2.0 \pm 0.2 \mathrm{~mm}$ thicknesses, both in the presence and absence of ambient light $(p<0.001)$. The analysis of presence or absence of ambient light revealed statistically significant differences between mean oxygen saturation within the groups in which the thickness was $4.0 \pm 0.2 \mathrm{~mm}(<0.001), 3.0 \pm 0.2 \mathrm{~mm}$ $(<0.001)$ and $2.0 \pm 0.2 \mathrm{~mm}(<0.003)$.

In the positive control group, the mean oxygen saturation was $96.3 \%$, and the mean pulse rate was $69.5 \mathrm{bpm}$ in the presence of ambient light, in comparison with $96 \%$ and $70.5 \mathrm{bpm}$, respectively, in its absence. In the negative control group, no oxygen saturation or pulse rate values were recorded in any sample.

\section{Discussion}

The increase in the thickness of dental structures (enamel and dentin) from $2.0 \mathrm{~mm}$ to $4.0 \mathrm{~mm}$ revealed a significant reduction in oxygen saturation, from $96.4 \%$ to $92.7 \%$ in the presence of ambient light, and from $96.0 \%$ to $89.3 \%$ in its absence.

According to optical principles, when a light beam penetrates a medium that is not a vacuum, there is absorbance and loss of intensity and wave amplitude, thereby explaining the results of this study. ${ }^{11,23}$ The association between absorbance and thickness is direct, that is, the greater the thickness, the greater the absorbance. In contrast, the association with transmittance is inverse, namely, the smaller the thickness, the greater the light transmittance. ${ }^{8}$ In the case of enamel and dentin, when light propagates through them, there is diffraction in addition to absorbance. ${ }^{9,11}$ The enamel structure has hexagonal hydroxyapatite crystals organized into prisms. These prisms scatter the light that passes through them. ${ }^{11}$ Dentinal tubules seem to have an organization characterized by practically total reflection of all light beams in their entire extension. ${ }^{11}$

The increase in the thickness of the dental structure used in this study explains the significant decrease in oxygen saturation. The internal surface dimensions of dentin were reduced to keep light from reaching points

Table. Mean oxygen saturation value (\%) in each group according to thickness $(\mathrm{mm})$ of the interposed tooth structure and the presence or absence of light.

\begin{tabular}{|c|c|c|c|c|}
\hline \multirow{2}{*}{ Ambient light } & \multicolumn{3}{|c|}{ Dentin/enamel thickness (mm) } & \multirow{2}{*}{$p$-value } \\
\hline & $4.0 \pm 0.2$ & $3.0 \pm 0.2$ & $2.0 \pm 0.2$ & \\
\hline Present & $92.7 \% \pm 2.5^{a . A}$ & $95.5 \% \pm 1.3^{\mathrm{b} . \mathrm{A}}$ & $96.4 \% \pm 0.9^{c . A}$ & $<0.001$ \\
\hline Absent & $89.3 \% \pm 3.7^{a . B}$ & $94.5 \% \pm 2.0^{\mathrm{b} . \mathrm{B}}$ & $96.0 \% \pm 0.9^{c . B}$ & $<0.001$ \\
\hline p-value & $<0.001$ & $<0.001$ & $<0.003$ & \\
\hline
\end{tabular}

Different lowercase letters indicate statistically different means between groups. Different capital letters indicate statistically different means within groups. 
of different thicknesses on the same surface, otherwise requiring that one more variable be introduced in the analysis, while concomitantly preserving the external anatomy of the buccal surface of the tooth crown. The buccolingual angle of light incidence and the light position were similar to those used in the clinical pulse oximetry of the mandibular molars. The perpendicular incidence of light on the dentin might have added to the lower oxygen saturation values found in this study.

According to the optical properties of dental tissues, the emerging light flow has different values at each angle of incident light, insofar as these values are lower in the buccolingual than the occlusocervical dimension., ${ }^{3,9}$

Pulse oximetry depends on accurate measurements only obtainable in the range of $660-940 \mathrm{~nm}$, whereas the wavelengths generated by the light sources used in dental practices may fall out of this range, thus interfering with the accuracy of the device and consequent interpretation of its results. ${ }^{24}$ In this study, the pulse oximeter wavelengths used were $660 \mathrm{~nm}$ and $905 \mathrm{~nm}$. Red and infrared wavelengths used in medical pulse oximetry have been designed for tissues with a high volume of blood, such as the fingers. Therefore, they cannot be expected to be effective for the small volume of arterial blood in the pulp, since the amplitude of the arterial pulsatile/non-pulsatile component ratio is low. ${ }^{25} \mathrm{Schmidt}$ et al. ${ }^{26}$ analyzed the influence of wavelength on the detection of pulsatile pulp tissue variations in an in vitro blood circulation model using dental (25) analyzed the interference of wavelength on the detection of pulsatile variations of the pulp tissue in an in vitro model of blood circulation using tooth plethysmography, and concluded that the ideal visible spectrum wavelength for measurement in teeth is between $540-580 \mathrm{~nm}$.

In pulse oximetry measurements, light transmittance through hemoglobin is determined by the Beer-Lambert law. ${ }^{27}$ This law can be used to calculate the value of oxygen saturation in the blood, and provides an approximate value of the actual oxygen saturation. According to this law, incident light equals the sum of light absorbance and transmittance, but does not consider light reflectance and diffraction, which may interfere with the results of pulse oximetry, when this oximetry is used to evaluate teeth, because of the presence of enamel and dentin. Moreover, variations of area thickness and volume are not taken into consideration when using a medical oximeter. ${ }^{27}$

Reported age-related changes in dentin structure ${ }^{28,29}$ seem to interfere in the results of pulse oximetry. ${ }^{18}$ The continuous secretion and mineralization of the dentin matrix by odontoblasts increases the mean dentin thickness of all teeth by $17.1 \%$ in the crown. ${ }^{27}$ Significant differences were found in the diameter of dentinal tubules of young individuals' teeth, compared with that of older individuals. The dentin of young teeth has dentinal tubules with open lumens, whereas the tubules of older individuals are completely filled with minerals. ${ }^{29,30}$ Therefore, the dentin of younger individuals may have optical properties different from those of older persons. Mineral depositions during aging lead to a gradual narrowing of the pulp circumference, and consequent decrease in the size of the pulp chamber, and the number of capillaries that nourish odontoblasts and predentin. ${ }^{31}$ These changes are attributed to the remodeling of the pulp tissue to accommodate the narrowing of the pulp chamber. ${ }^{28}$

Although this study used extracted teeth, the tubule lumen samples were kept hydrated during the entire experiment by filling them continuously with liquid. Walton et al. ${ }^{6}$ found that light propagated better in disks of hydrated versus dehydrated dentin. Replacing water with air causes changes attributed to the difference in the refractive index. Therefore, since the index for air is 1.0 and that of water is 1.33, which is closer to the 1.53 dentin index ${ }^{3}$ the hydration of the tooth structure must be adjusted when the pulse oximetry is measured.

The results of this in vitro study should not be extrapolated directly to in vivo conditions, since several factors may affect light transmittance, and there are inherent differences between finger and dentin vascularization. In a vital tooth, dentinal tubules are not empty, since the odontoblast process occurs in the tubular compartment, filled with tissue fluid, and lined by an organic sheath. Lima et al..$^{32}$ used the findings of a randomized clinical trial to assess pulp oxygen saturation $\left(\mathrm{SaO}_{2}\right)$ in maxillary central incisors after dental bleaching. Pulp $\mathrm{SaO}_{2}$ in maxillary central incisors was similar at baseline, and decreased 
immediately after in-office bleaching, regardless of the use of desensitizing toothpaste, but increased at 30 days after dental bleaching. Thus, monitoring pulp oxygen saturation levels before, during, and after tooth bleaching is an essential procedure.

Ambient light does not seem to affect pulse oximetry results. This finding can be explained by the principles of plethysmography used to detect arterial pulse and measure oxygen saturation of arterial blood. ${ }^{24}$ Photodetectors, which collect light 480 times per second, can measure ambient light during both the static and pulsatile phase. Pulse oximetry technology does not seem to take into consideration tissue, venous blood, or incident energy from sources in the environment. ${ }^{24}$ In this study, there were significant differences in the mean oxygen saturation levels in the presence or absence of ambient light, when the dental surfaces were interposed, thus suggesting that the light-emitting diode should be covered to protect it from ambient light. The results of this study corroborate those reported by Amar et al., ${ }^{33}$ who found that fluorescent light interfered with the interpretation of pulse oximetry results.

Currently, pulse oximetry is a well-established resource in medicine, as a regular clinical procedure during anesthesia. The dental pulp is vascularized, just like the ear lobe and the finger. Therefore, pulse oximetry can measure oxygen saturation to assess pulp vitality.

In this study, patient movements, lack of parallel alignment and the interference caused by the pulsation of the operator's finger were eliminated by a device specially designed to position the sensors. Light transmittance sensors were kept at a distance, determined by tooth diameter. The distance between the light emitting source and the photodiode should be the shortest possible to maximize the amount of light transmitted.

Nevertheless, despite the ongoing technological advances of pulse oximeters ever since they were invented, and the introduction of reflective sensors, which eliminate the interference caused by the distance between sensors, oximeters still must be downsized, so that they can be used on different surfaces, such as teeth. ${ }^{34}$ Further studies should be conducted to elucidate the mechanism of light and dentinal tubule interaction to professionals.

In summary, enamel and dentin thickness interfered with the level of oxygen saturation, regardless of the presence or absence of ambient light. Oxygen saturation levels were lower for thicker enamel and dentin samples.

\section{References}

1. Estrela C, Holland R, Estrela CR, Alencar AH, Sousa-Neto MD, Pécora JD. Characterization of successful root canal treatment. Braz Dent J. 2014 Jan-Feb;25(1):3-11. https://doi.org/10.1590/0103-6440201302356

2. Krishna VG, Kandaswamy D, Gupta T. Assessment of the efficacy of an indigeniously developed pulse oximeter dental sensor holder for pulp vitality testing. Indian J Dent Res. 2006 Jul-Sep;17(3):111-3. https://doi.org/10.4103/0970-9290.29880

3. Vaarkamp J, ten Bosch JJ, Verdonschot EH. Propagation of light through human dental enamel and dentine. Caries Res. 1995;29(1):8-13. https://doi.org/10.1159/000262033

4. Lighting BVP. Lighting manual. 5th ed. Holland: Eindhoven; 1993.

5. Spitzer D, Bosch JT. The absorption and scattering of light in bovine and human dental enamel. Calcif Tissue Res. 1975;17(2):129-37. https://doi.org/10.1007/BF02547285

6. Walton RE, Outhwaite WC, Pashley DF. Magnification: an interesting optical property of dentin. J Dent Res. 1976 Jul-Aug;55(4):639-42. https://doi.org/10.1177/00220345760550041601

7. O'Brien WJ. Fraunhofer diffraction of light by human enamel. J Dent Res. 1988 Feb;67(2):484-6. https://doi.org/10.1177/00220345880670021001

8. White JM, Lopes DP, Strawn SE, Marshall SJ, Johnston WM. Spectrophotometric absorbance and direct transmittance of dentin [abstract 1469]. J Dent Res. 1995;74:195.

9. Fried D, Glena RE, Featherstone JD, Seka W. Nature of light scattering in dental enamel and dentin at visible and near-infrared wavelengths. Appl Opt. 1995 Mar;34(7):1278-85. https://doi.org/10.1364/AO.34.001278

10. Vogel K, Salz U. Influence of trans-tooth curing on physical properties of composites [abstract 137]. J Dent Res. 1997;75:111. 
- Interference of coronal enamel and dentin thickness and ambient light on pulse oximetry interpretation

11. Brodbelt RH, O'Brien WJ, Fan PL, Frazer-Dib JG, Yu R. Translucency of human dental enamel. J Dent Res. 1981 Oct;60(10):1749-53. https://doi.org/10.1177/00220345810600100401

12. Calil E, Caldeira CL, Gavini G, Lemos EM. Determination of pulp vitality in vivo with pulse oximetry. Int Endod J. 2008 Sep;41(9):741-6. https://doi.org/10.1111/j.1365-2591.2008.01421.x

13. Anusha B, Madhusudhana K, Chinni SK, Paramesh Y. Assessment of pulp oxygen saturation levels by pulse oximetry for pulpal diseasesA diagnostic study. J Clin Diagn Res. 2017 Sep;11(9):ZC36-9. https://doi.org/10.7860/JCDR/2017/28322.10572

14. Hargreaves KM, Tay F, Goodis HE. Seltzer and Bender's dental pulp. 2nd ed. Chicago: Quintessence; 2012.

15. Schnettler JM, Wallace JA. Pulse oximetry as a diagnostic tool of pulpal vitality. J Endod. 1991 Oct;17(10):488-90. https://doi.org/10.1016/S0099-2399(06)81795-4

16. Ciobanu G, lon I, Ungureanu L. Testing of pulp vitality by pulsoximetry. Odontology. 2012;2:94-8.

17. Karayilmaz H, Kirzioglu Z. Comparison of the reliability of laser Doppler flowmetry, pulse oximetry and electric pulp tester in assessing the pulp vitality of human teeth. J Oral Rehabil. 2011 May;38(5):340-7. https://doi.org/10.1111/j.1365-2842.2010.02160.x

18. Estrela C, Serpa GC, Alencar AH, Bruno KF, Barletta FB, Felippe WT, et al. Oxygen saturation in the dental pulp of maxillary premolars in different age groups - Part 1. Braz Dent J. 2017 Jul-Sep;28(5):573-7. https://doi.org/10.1590/0103-6440201701660

19. Estrela C, Oliveira KS, Alencar AH, Barletta FB, Estrela CR, Felippe WT. Oxygen saturation in the dental pulp of maxillary and mandibular molars - Part 2. Braz Dent J. 2017 Nov-Dec;28(6):704-9. https://doi.org/10.1590/0103-6440201701447

20. Bruno KF, Barletta FB, Felippe WT, Silva JA, Alencar AHG, Estrela C. Oxygen saturation in the dental pulp of permanent teeth: a critical review. J Endod. 2014 Aug;40(8):1054-7. https://doi.org/10.1016/i.joen.2014.04.011

21. Setzer FC, Kataoka SH, Natrielli F, Gondim-Junior E, Caldeira CL. Clinical diagnosis of pulp inflammation based on pulp oxygenation rates measured by pulse oximetry. J Endod. 2012 Jul;38(7):880-3. https://doi.org/10.1016/i.joen.2012.03.027

22. Caldeira CL, Barletta FB, Ilha MC, Abrão CV, Gavini G. Pulse oximetry: a useful test for evaluating pulp vitality in traumatized teeth. Dent Traumatol. 2016 Oct;32(5):385-9. https://doi.org/10.1111/edt.12279

23. Jenkins FA, White HE. Fundaments of optics. 4th ed. Auckland: McGraw-Hill; 1981.

24. Fluck RR Jr, Schroeder C, Frani G, Kropf B, Engbretson B. Does ambient light affect the accuracy of pulse oximetry? Respir Care. 2003 Jul;48(7):677-80.

25. Kakino S, Kushibiki S, Yamada A, Miwa Z, Takagi Y, Matsuura Y. Optical measurement of blood oxygen saturation of dental pulp. ISRN Biomed Engineer. 2013;ID 502869. https://doi.org/10.1155/2013/502869

26. Schmitt JM, Webber RL, Walker EC. Optical determination of dental pulp vitality. IEEE Trans Biomed Eng. 1991 Apr;38(4):346-52. https://doi.org/10.1109/10.133229

27. Cerqueira MG. Desenvolvimento de um sensor de determinação da vitalidade dentária. Thesis (Doctoral). Coimbra: Faculdade de Ciências e Tecnologia, Universidade de Coimbra; 2013.

28. Murray PE, Stanley HR, Matthews JB, Sloan AJ, Smith AJ. Age-related odontometric changes of human teeth. Oral Surg Oral Med Oral Pathol Oral Radiol Endod. 2002 Apr;93(4):474-82. https://doi.org/10.1067/moe.2002.120974

29. Ryou H, Romberg E, Pashley DH, Tay FR, Arola D. Importance of age on the dynamic mechanical behavior of intertubular and peritubular dentin. J Mech Behav Biomed Mater. 2015 Feb;42:229-42. https://doi.org/10.1016/i.jmbbm.2014.11.021

30. Bernick S. Age changes in the blood supply to human teeth. J Dent Res. 1967 May-Jun;46(3):544-50. https://doi.org/10.1177/00220345670460031501

31. Stella JP, Barletta FB, Giovanella LB, Grazziotin-Soares R, Tovo MF, Felippe WT, et al. Oxygen Saturation in Dental Pulp of Permanent Teeth: Difference between Children/Adolescents and Adults. J Endod. 2015 Sep;41(9):1445-9. https://doi.org/10.1016/i.joen.2015.04.024

32. Lima LF, Alencar AHG, Decurcio DA, Silva JA, Favarão IN, Loureiro MA, et al. Effect of dental bleaching on pulp oxygen saturation in maxillary central incisors: a randomized clinical trial. J Appl Oral Sci. 2019;27:e20180442. https://doi.org/10.1590/1678-7757-2018-0442

33. Amar D, Neidzwski J, Wald A, Finck AD. Fluorescent light interferes with pulse oximetry. J Clin Monit. 1989 Apr;5(2):135-6. https://doi.org/10.1007/BF01617888

34. Kim J, Gutruf P, Chiarelli AM, Heo SY, Cho K, Xie Z, et al. Miniaturized Battery-Free Wireless Systems for Wearable Pulse Oximetry. Adv Funct Mater. 2017 Jan;27(1):1-8. https://doi.org/10.1002/adfm.201604373 